\title{
A Circularly Polarized Small-Size Microstrip Antenna using a Cross Slot with Enhanced Bandwidth \& Gain
}

\author{
Akshay Kumar ${ }^{1}$, Ekanshi Tanwer ${ }^{2}$, Anjali Singh ${ }^{3}$, Ankit kumar Saini ${ }^{4}$, \\ Mr. Ankur Gupta ${ }^{5}$ \\ (1, 2, 3, 4-Graduate students of Meerut Institute of Technology, Meerut- UP (India) \\ (5- Asstt. Prof.-Department of Electronics \& Communication, Meerut Institute of Technology-Meerut)
}

\begin{abstract}
In the recent years the development in communication systems requires the development of low cost, minimum weight, low profile antennas that are capable of maintaining high performance over a wide spectrum of frequencies. This technological trend has focused much effort on the design of a Microstrip patch antenna. A new, circularly polarized small-size microstrip antenna using a proximity coupled feed method is proposed. A simple configuration based on a cross slot with unequal slot lengths on a circular patch is adopted to realize a small-size element antenna. The measured results verify the circular polarization, and the antenna radius was reduced by about $22 \%$ by using the slot lengths which are nearly equal to the diameter of the circular patch antenna. Good impedance and axial ratio characteristics have been obtained.
\end{abstract}

\section{Introduction}

A circularly polarized antenna with a low profile, small size, and light weight is required in mobile satellite communications. Many types of microstrip antennas have been proposed and investigated [1].

Circularly polarized microstrip antennas are classified as single-fed type or dual-fed type, depending on the number of feed points necessary to generate the circularly polarized waves. The single-fed type has the advantage of not requiring an external polarizer such as a $90^{\circ}$ hybrid coupler. The relationship between the optimum probe location and the frequency of the obtained circularly polarized wave has been clarified, and good experimental results have been reported [2].Recently, aperture-coupled feed methods have been attracting much attention because their geometries are suitable for monolithic integration with microwave or milliwave devices. The feed position for a circularly polarized operation and the input impedance of several microstrip antennas fed by the slot coupled method have been investigated [3].However, with this type of microstrip antenna it is difficult to excite good circularly polarized waves.

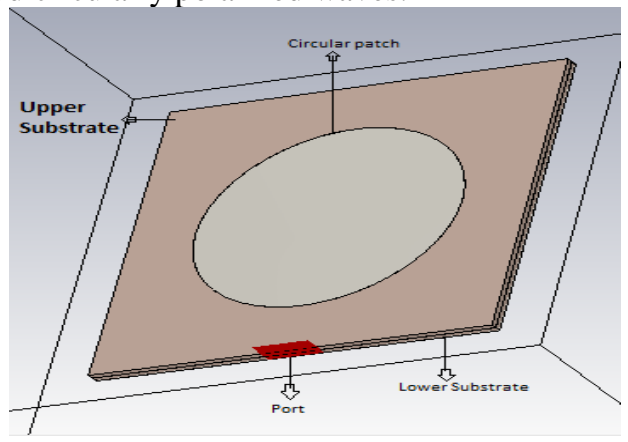

Fig.1.Structure Without Slot

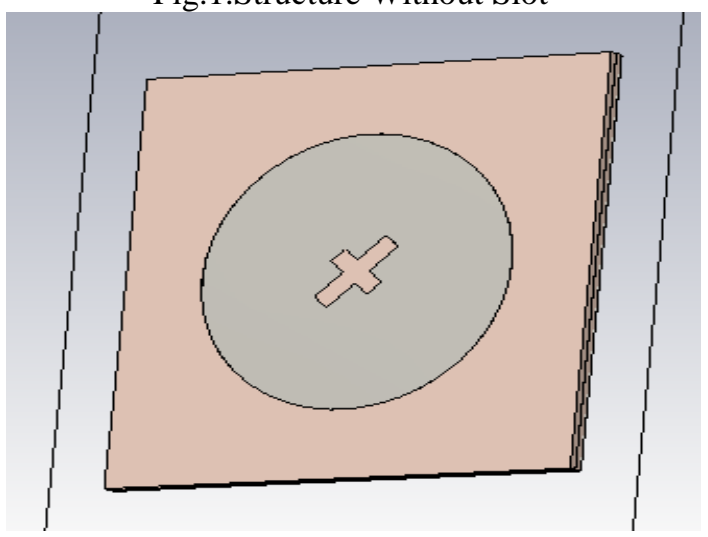

Fig.2Structure With Slot 


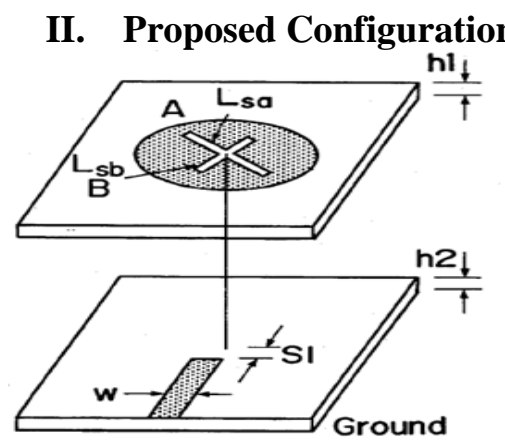

Fig. 3 Configuration of the Proposed Patch Antenna

Another suitable feed method is anelectromagneticallycoupled method which is also known as the proximity-coupled method. This type of antenna has several advantages overa directly fed patch antenna. By using a proximity-coupledmethod, an optimal feed point of amicrostrip antenna has beenproposed for linear polarization [4]. Moreover, a circularly polarized rectangularmicrostrip antenna, fed by proximity coupled method using an offset microstrip line, was proposed by the author [5]. On the other hand, the element of a phased-array antenna must be arranged at almost about half wavelength to obtain wide-angle beam scanning. The resonant frequency of a single fed circularly polarized microstrip antenna with a thin diagonal a patch antenna with a $90^{\circ}$ hybrid [6].Therefore, it is difficult to arrange an antenna element at about half wavelength in the case of the above-patch antenna. The purpose of this paper is to propose a small-size circular patch antenna using across slot with unequal slot lengths. The proposed antenna can achieve circular polarization without the need for a $90^{\circ}$ hybrid coupler. The measuredresultsare presented to demonstrate the usefulness of the proposed antennaconfiguration. Good impedance and axial ratio characteristics are realized.

\section{Antenna Configuration}

The proposed antenna configuration is shown in Fig. 1. Thecircular patch with a cross slot and the microstrip line are formed by the substrates with a dielectric constant $\boldsymbol{E}$ rand thickness hl and $h 2$, respectively. The radius of the circular patch is $\boldsymbol{r}$. Slot $\boldsymbol{A}$ with length $L s a$ and slot $B$ with length $L s b$, cross orthogonally at the center of each slot, which is the center of the circular patch. The characteristic impedance of the microstrip line is $50 \Omega$. Slis the distance between the end of the microstrip line and the center of the patch antenna.

\section{Experimental Results}

The resonant frequency of the linearly polarized circular patch with a slot can be controlled by changing the slot length [7]. The resonant frequency decreases monotonically with increasing slot length. Therefore, resonant frequencies of orthogonal modes, as a result of the perturbation caused by a cross slot, as shown in Fig. 1, will decrease with increasing slot lengths $L s a$ and $L s b$. Thus, the resonant frequency of the proposed patch antenna can be reduced. Consequently, the proposed antenna can be made small size and compact compared with a linearly polarized patch antenna with a single slot.The proposed antenna was designed and tested to verify circularly polarizing operation. The experimental models are madeof FR-4 Epoxy substrate with $\mathcal{E} \boldsymbol{r}=\mathbf{4 . 3}$ and the thickness $\boldsymbol{h} \boldsymbol{I}=\boldsymbol{h} \mathbf{2}=\mathbf{1 . 6} \mathrm{mm}$.

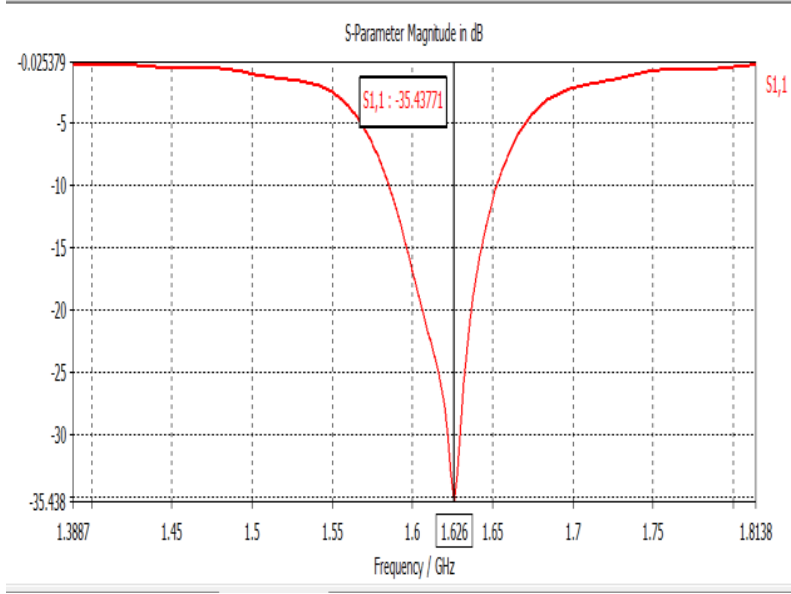

Fig.4 s-parameter 
The width of the microstrip line $\mathbf{W}$ was $4.5 \mathrm{~mm}$. The radius of the circular patch without a cross slot wasr= $33.31 \mathrm{~mm}$, and the resonant frequency was $\mathbf{1 . 6 4 6} \mathbf{G H z}$. The patch antenna was designed by using the simple cavity method[8] Fig. 2 shows the measured impedance and return loss for the Lsa $=20.0 \mathrm{~mm}$, Lsb= $12.0 \mathrm{~mm}$, and SI $=\mathbf{4 5 . 0} \mathrm{mm}$. Reference plane is the edge of the circular patch. Good impedance matching was obtained. The bandwidth VSWR less than 2 was4.08\%. The resonant frequency of the circular patch antenna without a cross slot was $1.626 \mathrm{GHz}$. Therefore, the antenna radius can be reduced by about $2 \%$ using the crossslot configuration compared with that of the circular patchwithout a slot.Fig.6 shows the measured axial ratios as a parameter of the slot length Lsb. A $0.98-\mathrm{dB}$ boresight axial ratio was obtained at $1.626 \mathrm{GHz}$ when Lsa $=20.0$ $\mathrm{mm}$ and $\mathrm{Lsb}=12.0 \mathrm{~mm}$. Fig.5 shows the measured gain was $7.4 \mathrm{dBi}$ at the boresight.To verify the possibility of achieving a reduction of the antenna size by using this proposed method, an antenna with Lsa $=50.0 \mathrm{~mm}$ and $\mathrm{Lsb}=36 \mathrm{~mm}$, nearly equal to the diameter of the circular patch antenna, was tested. Fig.3 shows the radiation pattem in the $1.071 \mathrm{GHz}$. An axial ratio of about $3.0 \mathrm{~dB}$ was obtained in the $\& 45^{\circ}$ range. Thus, the radius of the circular patch with a cross slot was reduced by about $22 \%$ compared with the radius of a circular patch without a cross slot. The gian was about $7.5 \mathrm{~dB}$, which is greater than that the theorical value of a probe-fed patch antenna without a cross slot.

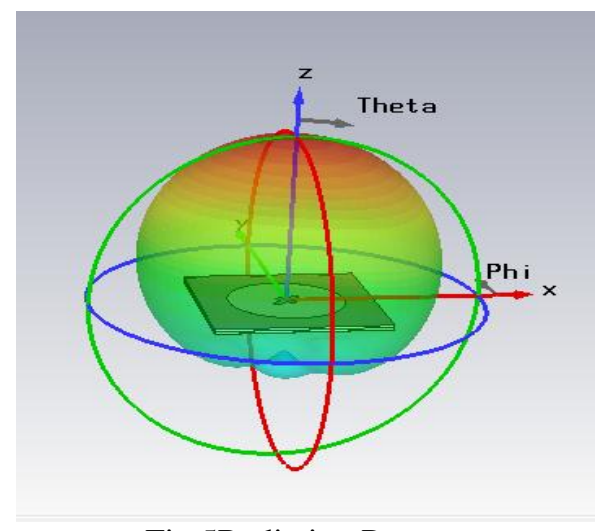

Fig.5Radiation Pattern

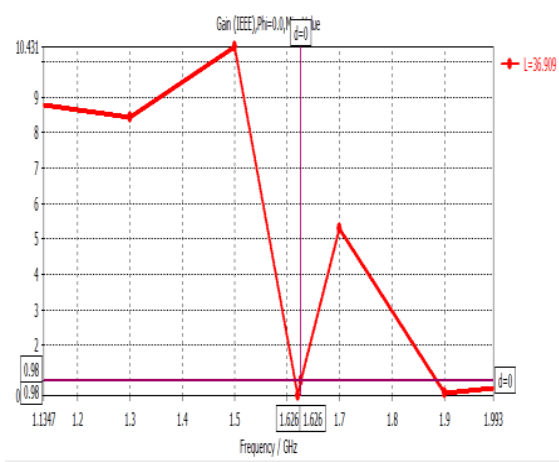

Fig.6Axail Ratio

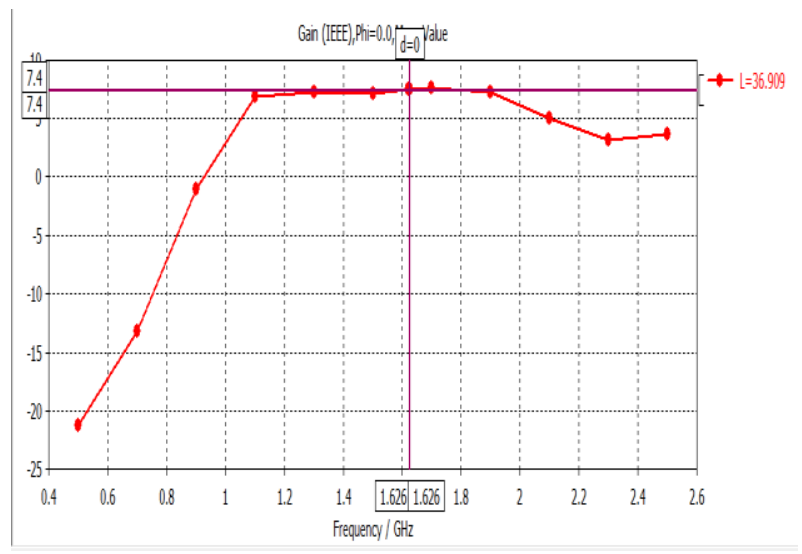

Fig.7Gain 


\section{Table}

COMPARISION TABLE OF VARIOUS SIMULATEDPARAMETERS.

Where,

\begin{tabular}{|l|l|l|l|l|}
\hline Lsa $(\mathrm{mm})$ & 20 & 30 & 40 & 50 \\
\hline Lsb(mm) & 12 & 20 & 28 & 36 \\
\hline $\mathrm{f}(\mathrm{GHz})$ & 1.626 & 1.626 & 1.626 & 1.626 \\
\hline $\mathrm{r}(\mathrm{mm})$ & 33.31 & 32.56 & 30.09 & 26.11 \\
\hline S11(dB) & -35.43 & -28.8 & -35 & -30 \\
\hline Gain(dB) & 7.6 & 7.3 & 7.39 & 7.5 \\
\hline AR(dB) & 0.98 & 2.36 & 2.78 & 0.98 \\
\hline fa(GHz) & 1.626 & 1.54 & 1.432 & 1.071 \\
\hline$\%$ BW & 4.40 & 3.51 & 3.98 & 4.08 \\
\hline
\end{tabular}

Lsa is length of slot A

Lsb is length of slot B

f is resonance frequency

$\mathbf{r}$ is radius of circular patch

S11 is return loss

AR is axial ratio

fa is frequency after varying the slot length.

\section{REDUCTION OF PATCH RADIUS WITH INCREASE INSLOT LENGTH}

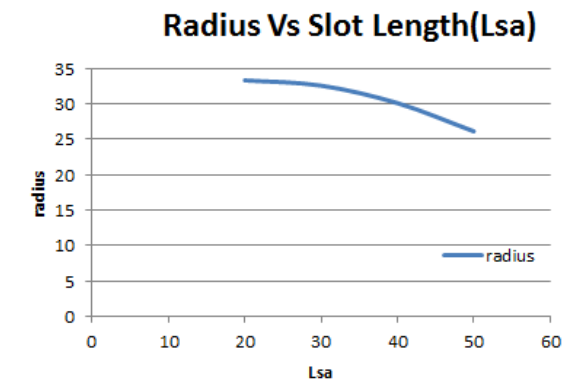

Radius Vs Slot Length(Lsb)

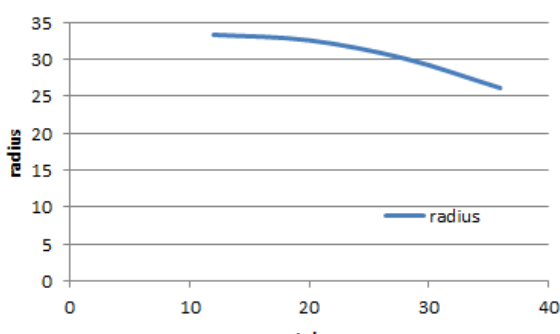

Lsb

REDUCTION OF RESONANCE FREQUENCY WITH INCREASE IN SLOT LENGTH

Variation of Frequency with Slot $A$

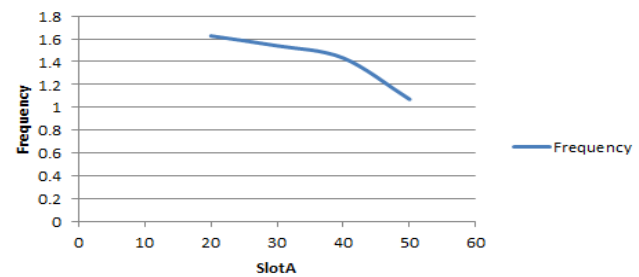

Variation of frequency with Slot B

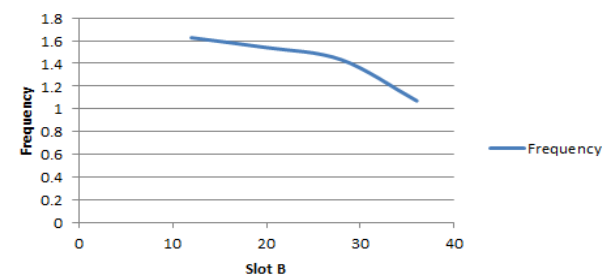


s-parameter magnitude in $\mathbf{d B}$

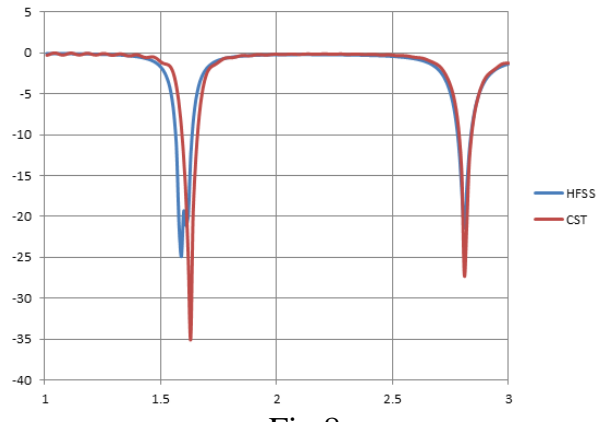

Fig.8

Fig.8 shows the simulated CST and simulated HFSS matching frequency band of the proposed antenna for dB reflection coefficient from $1.56 \mathrm{GHz}$ to $1.63 \mathrm{GHz}$ with a bandwidth of $\mathbf{4 . 6 1 \%}$ in simulation HFSS and the frequency band of $1.46 \mathrm{GHz}$ to $1.52 \mathrm{GHz}$ with a bandwidth of $\mathbf{4 . 0 8} \%$ in simulation CST respectively. We can observe the good agreement between the HFSS and CST simulated radiation patterns.

\section{Conclusion}

This paper describes the results of measurements of a new single-fed circularly poalrizedmicrostrip antenna. A small-size element antenna for circular polarization was realized by using a circular patch antenna with a cross slot having different arm lengths. The antenna radius was reduced by about $22 \%$ by of using slot lengths which are nearly equal to the diameter ofthe circular patch antenna. The proposed antenna is suitable for application in the field of mobile satellite communications as a phased-array antenna using a multilayered feed network integrated with microwavedevices.

\section{References}

[1] F. Naderi, "NASA'S mobile satellite communications program: Groundand space segment technologies," Los Angeles, CA, IAF84-84, 1984.

[2] Y. Suzuki, N. Miyano, and T. Chiba, "Circularly polarized radiationfrom singly fed equilateral-triangular microstripantenna," IEE Proc., vol. 134, pt. H, pp. 194-198, Apr. 1987

[3] W. H. Aksun, Z. H. Wang, S. L. Chung, and Y. T. Lo, "On slot-coupledmicrostrip antennas and their applications CP operation theory andexperiment,” IEEE Trans. Antennas Propagat., vol. 38, pp. 1224-1230,Aug. 1990.

[4] M. Davidovitz and Y. T. Lo, "Rigorous analysis of a circular patch antennaexcited by a microstrip transmission line," IEEE Trans. AntennasPropagat., vol. 37, pp. 949-958, Aug 1989.

[5] H. Iwasaki, H. Sawada, and K. Kawabata, "A circular polarized microstripantenna using singly-fed proximity coupled feed," in Proc. Int.Symp. Antennas Propagat., Sapporo, Japan, Sept. 1992, pp. 797-800.

[6] J. Ken, "Microstrip antenna developments," Workshop Antenna Technol.,New Mexico State Univ. Las Cruces, pp. 3.1-3.20, 1979.

[7] “Compact and Broadband MicrostripAntennas"Kin-Lu Wong, John Wiley \& Sons, 2002.

[8] HisaoIwasaki,IEEE Transactions On Antennas And Propagation. Vol.44,No.10,October 1996.

[9] T.F.Lai, Wan Nor Liza Mahadi, NorhayatiSoin, "Circular Patch Microstrip Array Antenna for KU-band" World Academy of Science, Engineering and Technology 48, 2008. [10]Veerendra Singh Jadaun, Pavan Kumar Sharma, AshishDuvey, "Design A Microstrip Patch Antenna of Single Band For 1.8GHz", international conference e-manthan 2012, 6-7 april 2012, Jhansi. 